\title{
Saccular Impairment in Alzheimer's Disease Is Associated with Driving Difficulty
}

\author{
Eric X. Wei ${ }^{a}$ Esther S. Oh ${ }^{b}$ Aisha Harun ${ }^{a}$ Matthew Ehrenburg ${ }^{a}$ \\ Yuri Agrawal ${ }^{a}$ \\ a Department of Otolaryngology - Head and Neck Surgery, Johns Hopkins University \\ School of Medicine, Baltimore, MD, USA; ${ }^{b}$ Department of Medicine, Johns Hopkins \\ University School of Medicine, Baltimore, MD, USA
}

\section{Keywords}

Alzheimer's disease - Vestibular system · Automobile driving - Spatial cognition - Saccule and utricle

\begin{abstract}
Background/Aims: Patients with Alzheimer's disease (AD) experience increased rates of vestibular loss. Recent studies suggest that saccular impairment in mild cognitive impairment $(\mathrm{MCl})$ and $A D$ patients is associated with impaired spatial cognitive function. However, the impact of saccular impairment on everyday behaviors that rely on spatial cognitive function is unknown. Methods: We recruited 60 patients (21 MCl and $39 \mathrm{AD}$ ) from an interdisciplinary Memory Clinic. Saccular function was measured, and a visuospatial questionnaire was administered to assess whether participants experienced impairments in terms of driving difficulty, losing objects, falls, and fear of falling. Results: In multiple logistic regression analyses, $\mathrm{MCl}$ and AD patients with bilateral saccular impairment had a significant, greater than 12 -fold odds of driving difficulty (OR $12.1,95 \% \mathrm{Cl} 1.2,117.7)$ compared to $\mathrm{MCl}$ and $\mathrm{AD}$ patients with normal saccular function, and the association appears to be mediated by spatial cognition as measured by the Money Road Map Test. Conclusion: This study suggests a novel link between saccular impairment and driving difficulty in $\mathrm{MCl}$ and $\mathrm{AD}$ patients and demonstrates that driving difficulty may be a real-world manifestation of impaired spatial cognition associated with saccular impairment.




\section{Introduction}

Patients with Alzheimer's disease (AD) commonly experience deficits in spatial cognition, such as spatial memory and navigation. Deficits in these skills have been shown to underlie many functional impairments in mild cognitive impairment (MCI) and AD patients. Several studies in $\mathrm{MCI}$ and $\mathrm{AD}$ patients have demonstrated that performance on spatial cognitive tests is a strong predictor of driving performance both in simulation and on-road conditions [1-3]. Additionally, spatial disorientation in MCI and AD has also been shown to contribute to falls risk [4-6], while deficits in spatial memory are thought to contribute to losing or misplacing objects [4, 7-9]. Longitudinal studies of MCI patients have shown that a subset of MCI patients develop severe visuospatial deficits [10], and this subset of MCI patients may be more likely to undergo clinical progression as determined by the Clinical Dementia Rating (CDR) scale over 2 years of follow-up [11].

The vestibular (inner ear balance) system plays an integral role in spatial memory and navigation abilities [12-16]. Vestibular ablation in rats results in impaired performance on foraging tasks requiring spatial memory and navigation [17-21]. Studies in humans have demonstrated that vestibular loss leads to impaired performance on neurocognitive tests of spatial memory and navigation tasks and also leads to hippocampal atrophy, which may mediate the link between vestibular and spatial cognitive function $[16,22,23]$. Patients with $\mathrm{AD}$ have been shown to have greater rates of vestibular loss compared to age-matched controls [24-26]. In recent work, we showed that AD patients with vestibular loss, specifically loss of saccular function, are significantly more likely to have spatial cognitive deficits (measured by neurocognitive tests of spatial cognition) relative to AD patients with normal vestibular function [27]. However, it is unknown whether saccular impairments may be related to real-world behaviors indicative of spatial cognitive deficits in MCI and AD patients.

In this study, we evaluated 60 patients with MCI and AD who were seen in the Johns Hopkins Memory and Alzheimer's Treatment Center (JHMATC), an interdisciplinary memory disorder clinic, and the Johns Hopkins Alzheimer's Disease Research Center (JHADRC). We assessed whether saccular impairment was associated with behavioral symptoms related to impaired spatial cognition, specifically losing objects, driving difficulty, and falls. Our study provides evidence that saccular impairment contributes to impaired driving ability and demonstrates a real-world behavioral correlate of the role of vestibular function in spatial cognition in MCI and AD patients.

\section{Methods}

\section{Study Participants}

This was a single-center prospective cross-sectional study. Participants were recruited from December 2014 until August 2017 from the JHMATC, an interdisciplinary memory disorder clinic, and the JHADRC. Inclusion criteria for the study were (1) age $\geq 55$ years; (2) diagnosis of MCI or AD; (3) Mini-Mental State Examination (MMSE) score $\geq 11$; (4) fluency in English; and (5) ability to obtain informed consent from the participant or a legally authorized representative. A diagnosis of MCI or AD was made using the National Institute on Aging-Alzheimer's Association diagnostic criteria [28, 29]. An MMSE score cutoff of $\geq 11$ was used as it has been shown to correspond to the threshold between moderate and severe dementia [30]. Prior to participation, a Memory Center physician determined the participant's ability to follow examination procedures.

Patients were excluded from the study if they had a previous history of vestibular disease or were unable to understand and participate in examination procedures. Demographic characteristics (age, gender, and education) were obtained from the patients' charts. Education was classified as less than college, college, or greater than college. All participants provided written informed consent. The study was approved by the Johns Hopkins Institutional Review Board. 


\begin{tabular}{l|l}
\hline Dement Geriatr Cogn Disord 2017;44:294-302 \\
\hline DOI: 10.1159/000485123 & $\begin{array}{l}\text { @ 2018 S. Karger AG, Basel } \\
\text { www.karger.com/dem }\end{array}$ \\
\hline
\end{tabular}

Wei et al.: Saccular Impairment in Alzheimer's Disease Is Associated with Driving Difficulty

\section{Saccular Function Tests}

Saccular function was assessed with the sound-evoked cervical vestibular evoked myogenic potential (cVEMP). Recordings were made with a commercial electromyographic system (software version 21.1; Natus Neurology, Middletown, WI, USA), with self-adhesive electrodes from GN Otometrics (Schaumburg, IL, USA). Testing methods have been published in detail previously and are described briefly here [31-33]. Participants sat on a chair with their heads inclined at $30^{\circ}$ from the horizontal. Trained examiners placed recording electromyographic electrodes on the sternocleidomastoid (SCM) muscle and the sternoclavicular junction bilaterally. A ground electrode was placed on the manubrium sterni. Patients were instructed to turn their heads against resistance to activate the SCM muscle. A sweep of auditory tone bursts (500 Hz, $125 \mathrm{~dB}$ SPL) was delivered monaurally through headphones (Viasys Healthcare, Madison, WI, USA), and inhibitory potentials were recorded from the ipsilateral SCM muscle. The presence or absence of a cVEMP response was recorded for each ear, indicating normal or impaired saccular function, respectively, as characterized by published guidelines [31-33].

\section{Visuospatial Questionnaire}

Given increasing evidence linking vestibular loss, including saccular impairment, to impairments in spatial cognition $[12,16,23]$, we sought to evaluate the association between saccular function and familyand patient-reported difficulties with 3 behavioral symptoms that reflect spatial cognition: losing objects [4, 7-9], difficulty driving [1-3], and falls [4-6]. As an additional measure of fall risk, we also asked about fear of falling. At present, no validated questionnaires probing visuospatial skills in MCI/AD patients have been published; therefore, we devised 4 simple questions. Participants and/or family members were asked the following questions in person or via telephone:

1. Do you (or the care recipient) lose objects? Yes or no.

2. Do you (or the care recipient) have difficulty driving? Yes or no.

3. Have you (or the care recipient) ever fallen? Yes or no.

4. Are you (or the care recipient) afraid of falling? Yes or no.

\section{Money Road Map Test}

We administered the Money Road Map Test (MRMT), a measure of visuospatial ability [34-36], to assess whether any potential associations between saccular impairment and behavioral symptoms may be mediated by spatial cognitive impairment. We previously reported that saccular impairment in MCI and AD patients was associated with spatial cognitive impairment, as determined by errors on MRMT [27]. The procedure has been published in detail [34] and will be briefly explained here. The participant was shown a 2D representation of a small city map on a sheet of paper in a fixed orientation. The city map contains a walking path with 32 turns. The participant was asked to imagine that they were traveling along the route through the city. As the examiner traced the route, the examiner asked the participant at each turn whether a right or left turn would be required to continue along the route. The participant's responses were recorded at each turn by the examiner up until a 300-s time limit. The main outcome of interest was the number of errors (i.e., incorrect or missing responses) out of 32 turns. Among the sample of 60 participants, a subsample of 48 (19 MCI and $29 \mathrm{AD}$ ) were able to complete the MRMT. Twelve patients (2 MCI, $10 \mathrm{AD}$ ) were not able to complete the MRMT because of inability to understand the test procedures after the instructions were explained once prior to the start of testing and up to 2 additional times.

\section{Statistical Analysis}

Descriptive statistics were conducted to evaluate participant demographics, saccular function, responses to visuospatial questionnaire items, and performance on the MRMT by category of cognitive impairment (MCI and AD).

We evaluated the relationship between each visuospatial questionnaire item and saccular function in multivariable analyses. Saccular function was categorized as normal function, unilaterally absent function, or bilaterally absent function. Responses to each questionnaire item were categorized with 0 representing "no" and 1 representing "yes." Multiple logistic regression models adjusting for demographic characteristics (age, sex, and education) were developed to explore the association between saccular impairment and each item on the visuospatial questionnaire. Furthermore, to evaluate whether the association between saccular function and driving difficulty was mediated by spatial cognition, we added the number of errors on MRMT to the regression model as a proxy for spatial cognition. All analyses were performed using STATA version 14 (College Station, TX, USA). 
Dementia

Cognitive Disorders

Table 1. Demographic characteristics of the study population, saccular function, and responses on the visuospatial questionnaire

\begin{tabular}{l|l}
\hline \multicolumn{2}{l}{ Dement Geriatr Cogn Disord 2017;44:294-302 } \\
\hline DOI: 10.1159/000485123 & $\begin{array}{l}\text { C 2018 S. Karger AG, Basel } \\
\text { www.karger.com/dem }\end{array}$ \\
\hline
\end{tabular}

Wei et al.: Saccular Impairment in Alzheimer's Disease Is Associated with Driving Difficulty

\begin{tabular}{|c|c|c|}
\hline & $\begin{array}{l}\text { MCI } \\
(n=21)\end{array}$ & $\begin{array}{l}\mathrm{AD} \\
(n=39)\end{array}$ \\
\hline \multicolumn{3}{|l|}{ Sex } \\
\hline Male & $9(42.9)$ & $10(25.6)$ \\
\hline Female & $12(57.1)$ & $29(74.4)$ \\
\hline Age, years & $71.5 \pm 9.7$ & $76.3 \pm 6.5$ \\
\hline \multicolumn{3}{|l|}{ Education } \\
\hline Less than college & $6(28.6)$ & $12(30.8)$ \\
\hline College & $6(28.6)$ & $20(51.3)$ \\
\hline Greater than college & $9(42.9)$ & $7(17.9)$ \\
\hline \multicolumn{3}{|l|}{ Saccular function } \\
\hline Normal function & $7(33.3)$ & $4(10.3)$ \\
\hline Unilateral loss & $7(33.3)$ & $11(28.2)$ \\
\hline Bilateral loss & $7(33.3)$ & $24(61.5)$ \\
\hline \multicolumn{3}{|l|}{ Visuospatial questionnaire } \\
\hline Lose objects ${ }^{\mathrm{a}}$ & $12(57.1)$ & $27(69.2)$ \\
\hline Driving difficulty ${ }^{\mathrm{b}}$ & $5(23.8)$ & $20(51.3)$ \\
\hline Falls $\mathrm{s}^{\mathrm{c}}$ & $8(38.1)$ & $15(38.5)$ \\
\hline Fear of falling ${ }^{\mathrm{d}}$ & $6(28.6)$ & 7 (17.9) \\
\hline MRMT errors & $4.5 \pm 6.2^{\mathrm{e}}$ & $11.0 \pm 6.6^{\mathrm{f}}$ \\
\hline
\end{tabular}

Values are means \pm standard deviations or $n(\%)$. MCI, mild cognitive impairment; AD, Alzheimer's disease; MRMT, Money Road Map Test. a Do you (or the care recipient) lose objects? ${ }^{\text {b }}$ Do you (or the care recipient) have difficulty driving? ${ }^{c}$ Have you (or the care recipient) ever fallen? ${ }^{\mathrm{d}}$ Are you (or the care recipient) afraid of falling? ${ }^{\mathrm{e}}$ Nineteen MCI patients completed the MRMT. ${ }^{\mathrm{f}}$ Twenty-nine AD patients completed the MRMT.

\section{Results}

Sixty patients (21 MCI and $39 \mathrm{AD}$ ) completed cVEMP testing and the visuospatial questionnaire (Table 1). AD patients (mean age of 76.3 years, SD 6.5) were slightly older than MCI patients (mean age of 71.5 years, SD 9.7). Compared to AD patients, MCI patients were less likely to report difficulty with losing objects (57.1\% in MCI and $69.2 \%$ in AD), driving difficulty (23.8\% in MCI and 51.3\% in AD), and falls (38.1\% in MCI and 38.5\% in AD) but more likely to report being afraid of falls $(28.6 \%$ in $\mathrm{MCI}$ and $17.9 \%$ in $\mathrm{AD})$. AD patients made a greater number of errors on the MRMT (mean 11.0, SD 6.6) compared to MCI patients (mean 4.5, SD 6.2).

Multiple logistic regression analyses were conducted to assess the association between saccular impairment and each questionnaire item in MCI and AD patients (Table 2). Compared to patients with normal saccular function, patients with unilateral saccular impairment had a nonsignificant increased odds of experiencing driving difficulty (OR 6.9, 95\% CI 0.7, 72.6), while patients with bilateral saccular impairment had a significant, over 12-fold odds of experiencing driving difficulty (OR 12.1, 95\% CI 1.2, 117.7). We did not observe any significant difference in the odds of losing objects, falling, or fear of falling by level of saccular function.

To assess whether the association between saccular impairment and driving difficulty may be mediated by spatial cognitive impairment, errors on MRMT was added to the regression model for driving difficulty as a proxy for spatial cognition. The addition of MRMT performance in the model for driving difficulty substantially reduced the coefficients for unilateral and bilateral saccular impairment in the model, and bilateral saccular impairment 
Table 2. Saccular impairment and visuospatial functional impairments

\begin{tabular}{|c|c|c|c|c|c|c|c|c|}
\hline & \multicolumn{2}{|l|}{ Losing objects } & \multicolumn{2}{|l|}{ Driving difficulty } & \multicolumn{2}{|l|}{ Falls } & \multicolumn{2}{|l|}{ Fear of falling } \\
\hline & OR $(95 \% \mathrm{CI})$ & $p$ value & OR $(95 \% \mathrm{CI})$ & $p$ value & OR $(95 \%$ CI $)$ & $p$ value & OR $(95 \% \mathrm{CI})$ & $p$ value \\
\hline \multicolumn{9}{|l|}{ Saccular impairment } \\
\hline Normal function & 1.0 (ref.) & & 1.0 (ref.) & & 1.0 (ref.) & & 1.0 (ref.) & \\
\hline Unilateral loss & $3.1(0.6,16.2)$ & 0.19 & $6.9(0.7,72.6)$ & 0.11 & $0.8(0.1,4.2)$ & 0.79 & $2.9(0.3,32.8)$ & 0.39 \\
\hline Bilateral loss & $2.5(0.6,11.3)$ & 0.23 & $12.1(1.2,117.7)$ & 0.03 & $0.8(0.2,3.4)$ & 0.71 & $4.1(0.4,41.0)$ & 0.23 \\
\hline Age & $1.0(0.9,1.1)$ & 0.86 & $1.0(0.9,1.1)$ & & $1.0(0.9,1.1)$ & 0.87 & $1.0(0.9,1.1)$ & 0.87 \\
\hline \multicolumn{9}{|l|}{ Sex } \\
\hline Male & 1.0 (ref.) & & 1.0 (ref.) & & 1.0 (ref.) & & 1.0 (ref.) & \\
\hline Female & $0.8(0.2,2.9)$ & 0.76 & $2.1(0.5,8.5)$ & 0.28 & $2.6(0.7,9.9)$ & 0.15 & $0.7(0.2,3.3)$ & 0.70 \\
\hline \multicolumn{9}{|l|}{ Education } \\
\hline Less than college & 1.0 (ref.) & & 1.0 (ref.) & & 1.0 (ref.) & & 1.0 (ref.) & \\
\hline College & $2.0(0.5,7.6)$ & 0.31 & $2.4(0.6,9.5)$ & 0.22 & $0.5(0.1,1.6)$ & 0.23 & $0.7(0.2,2.9)$ & 0.60 \\
\hline Greater than college & $1.2(0.3,5.7)$ & 0.80 & $0.4(0.1,2.6)$ & 0.35 & $0.4(0.1,1.8)$ & 0.21 & $0.3(0.04,2.1)$ & 0.21 \\
\hline
\end{tabular}

Italics indicate statistical significance. OR, odds ratio; $\mathrm{CI}$, confidence interval.

Table 3. Association between saccular impairment and driving adjusting for spatial cognition

\begin{tabular}{lll}
\hline & Driving & \\
\cline { 2 - 3 } & OR $(95 \% \mathrm{CI})$ & $p$ value \\
\hline $\begin{array}{l}\text { Saccular impairment } \\
\quad \text { Normal function }\end{array}$ & 1.0 (ref.) & \\
$\quad$ Unilateral loss & $2.8(0.2,35.3)$ & 0.42 \\
$\quad$ Bilateral loss & $4.0(0.3,48.2)$ & 0.28 \\
Age & $1.0(0.9,1.1)$ & 0.58 \\
Sex & $1.0($ ref.) & \\
$\quad$ Male & $1.5(0.3,8.1)$ & 0.64 \\
$\quad$ Female & $1.0($ ref. $)$ & \\
Education & $3.1(0.6,16.6)$ & 0.18 \\
$\quad$ Less than college & $1.1(0.1,9.4)$ & 0.91 \\
$\quad$ College & $1.1(0.9,1.2)$ & 0.30 \\
$\quad$ Greater than college & & \\
Errors on MRMT & & \\
\hline
\end{tabular}

OR, odds ratio; CI, confidence interval; MRMT, Money Road Map Test.

was no longer significantly associated with driving difficulty. Specifically, after adjusting for MRMT performance, patients with unilateral saccular impairment had a 2.8 odds ( $95 \%$ CI 0.2 , $35.3)$ and patients with bilateral saccular impairment had a 4.0 odds (95\% CI $0.3,48.2)$ of experiencing driving difficulty relative to patients with normal saccular function (Table 3).

\section{Discussion}

In this study of MCI and $\mathrm{AD}$ patients, bilateral saccular impairment, as determined by bilaterally absent responses on the sound-evoked cVEMP, was significantly associated with a greater than 12-fold odds of driving difficulty, while unilateral saccular impairment had a borderline nonsignificant nearly 7-fold odds of driving difficulty. Moreover, the associations 
Wei et al.: Saccular Impairment in Alzheimer's Disease Is Associated with Driving Difficulty

between saccular impairment and driving difficulty appear to be mediated by performance on the MRMT, a measure of spatial cognition. These findings suggest a novel link between saccular impairment and a real-world consequence of impaired spatial cognition in MCI and AD patients: driving difficulty.

Our study in MCI and AD patients builds on prior studies that also observed a link between vestibular dysfunction and driving difficulty in cognitively intact individuals with vestibular disorders. Studies in patients with specific vestibular disorders found that $60 \%$ of patients with Ménière disease found driving to be either difficult or dangerous to perform [37], and $30 \%$ of patients with vestibular schwannomas experienced driving difficulty following surgical resection of their tumors [38]. Recently published data from the National Health and Nutrition Examination Survey (NHANES) found that individuals with symptomatic vestibular dysfunction had an over 4-fold odds of experiencing driving difficulty relative to individuals without vestibular dysfunction [39]. Furthermore, when assessing specific driving conditions, 1 study found that vestibular patients have particularly greater difficulty with driving tasks that require spatial navigation, such as changing lanes in traffic, staying in lane, and pulling into or out of parking spaces, as well as with driving tasks where vision was limited, such as driving at night or in the rain [40]. On-road studies have further demonstrated that patients with bilateral vestibular loss exhibit reduced accuracy in reading and processing signs while sitting in a car [41] and slower horizontal head movements during driving [42]. Our study builds on previous work in patients with vestibular disorders by extending the association between saccular impairment and driving difficulty to $\mathrm{MCI}$ and $\mathrm{AD}$ patients. These findings are particularly relevant given that patients with MCI and AD have long been shown to have greater impairments in driving ability and $\mathrm{AD}$ drivers have a 2.3-fold increased risk of crash involvement compared to age-matched controls [1, 3, 43-45].

Our finding that the association between saccular impairment and driving difficulty in $\mathrm{MCI}$ and $\mathrm{AD}$ patients appears to be mediated by spatial cognition further suggests that driving difficulty may be a consequence of the contribution of the saccule to spatial cognitive function. Neuropsychological studies have established that spatial cognitive function, as determined by measures such as the Intersecting Pentagon Copying, Clock-Face Drawing, and Block Design tests, predicts driving performance and crash involvement in MCI and AD patients, and in older adults more broadly [46-52]. Meanwhile, a growing body of literature has demonstrated that vestibular function significantly contributes to performance on spatial cognitive measures. Animal studies have shown that rodents with absent vestibular function exhibit impaired performance on behavioral tasks of spatial memory and navigation [17-20] and also exhibit loss of location-specific firing in hippocampal place cells [15, 21]. These observations were corroborated by human studies which demonstrated that vestibular input, particularly saccular function, mediates performance on static and dynamic assessments of spatial navigation and memory [12-14], including the Triangle Completion Task (TCT) [16, 22]. Moreover, we observed in recent work that MCI and AD patients with saccular impairment, as determined by cVEMP responses, were significantly more likely to have spatial cognitive impairment relative to $\mathrm{MCI}$ and $\mathrm{AD}$ patients with normal vestibular function [27]. The hippocampus is considered the anatomic locus of spatial cognition [53] and, indeed, has been shown to receive substantial input from the vestibular system. Functional neuroimaging studies have demonstrated that saccular stimulation (specifically, sound-evoked cVEMP stimulation) activates the hippocampus [54], while patients with bilateral vestibular loss exhibited reduced hippocampal volume with concomitant poorer performance on a virtual navigation task compared to age-matched controls [23]. Our study thus builds on previous work establishing that vestibular dysfunction, and specifically saccular impairment, contributes to spatial cognitive impairment, by presenting driving difficulty as a real-world behavioral correlate of this relationship in MCI and AD patients. 
Wei et al.: Saccular Impairment in Alzheimer's Disease Is Associated with Driving Difficulty

In our study, saccular impairment was not significantly associated with losing objects, falls, and fear of falling. Losing objects may reflect higher-order cognitive processes in addition to visuospatial skills, such as attention [4], while falls risk may represent a more complex interaction of multiple factors, such as medication use, vision, bone and muscle strength, reaction time, and general cognitive ability [5,55-58]. Further prospective studies in larger samples will be needed to clarify the relationship between saccular function and these as well as other behavioral correlates of impaired spatial cognition in $\mathrm{MCI}$ and $\mathrm{AD}$, such as wandering [59].

We note important limitations in our study. The study was cross-sectional and, thus, cannot support causal inferences between saccular impairment and driving difficulty in MCI and $\mathrm{AD}$ patients. We acknowledge the potential for reverse causality or, alternatively, that other factors, such as traumatic brain injury, hypertension, diabetes, and/or cardiovascular disease, may contribute to both saccular impairment and spatial cognitive decline [26]. Nevertheless, our findings can be interpreted in the context of previous studies that suggest a causal contribution of saccular impairment to spatial cognitive dysfunction, especially given that the association between saccular impairment and driving difficulty in this study appears to be mediated by spatial cognition. Moreover, although we elected to focus on cVEMPs in the current study, further studies employing other measures of vestibular function may be valuable. An additional limitation of the study is that we did not differentiate between subtypes of MCI, which may or may not be of likely AD cause. Further work focused on driving difficulty and spatial cognition in MCI patients could restrict the study sample to MCI patients with likely AD etiology.

In summary, in this study of MCI and AD patients, a significant association was found between bilateral saccular impairment and self-reported difficulty driving a motor vehicle, and this association appears to be mediated by spatial cognition. This finding suggests that driving difficulty may be a real-life behavioral manifestation of the role of saccular impairment on impaired spatial cognition in $\mathrm{MCI}$ and $\mathrm{AD}$ patients. This study may have important clinical implications as difficulty driving is a challenging condition in patients with MCI and AD, who as a result face driving-related morbidity and reduced autonomy. Identifying a risk factor for driving difficulty, such as saccular impairment, which is potentially modifiable through vestibular rehabilitation, could be of significant benefit to MCI and AD patients. Clinicians seeing $\mathrm{MCI}$ and $\mathrm{AD}$ patients who report a history of driving impairments or accidents should consider diagnosis and treatment of potential underlying vestibular dysfunction. Further investigations are needed to evaluate whether vestibular rehabilitation may improve driving ability in MCI and AD patients who report difficulty driving a motor vehicle.

\section{Acknowledgments}

E.X.W. was a participant in the Medical Student Training in Aging Research program, funded by the American Federation for Aging Research and the National Institute on Aging (NIA). E.S.O. was funded by a NIA/NIH K23 Award (1K23AG043504-01), the Roberts Gift Fund, and the Ossoff Family Fund. A.H. was funded by a NIH T32 Award (5T32DC000027-25) and an AAO-HNSF Core Grant (349386). Y.A. was funded by a NIH K23 Award (5K23DC013056-02). The Alzheimer's Disease Research Council is supported by an NIA award (3P50AG005146-32S1).

\section{Disclosure Statement}

The authors have no conflicts of interest to report. 
Wei et al.: Saccular Impairment in Alzheimer's Disease Is Associated with Driving Difficulty

\section{References}

1 Hird MA, Egeto P, Fischer CE, Naglie G, Schweizer TA, Pachana N: A systematic review and meta-analysis of on-road simulator and cognitive driving assessment in Alzheimer's disease and mild cognitive impairment. J Alzheimers Dis 2016;53:713-729.

2 Aksan N, Anderson SW, Dawson J, Uc E, Rizzo M: Cognitive functioning differentially predicts different dimensions of older drivers' on-road safety. Accid Anal Prev 2015;75:236-244.

3 Ott BR, Daiello LA: How does dementia affect driving in older patients? Aging Health 2010;6:77-85.

4 Liu L, Gauthier L, Gauthier S: Spatial disorientation in persons with early senile dementia of the Alzheimer type. Am J Occup Ther 1991;45:67-74.

5 Taylor ME, Delbaere K, Lord SR, Mikolaizak AS, Brodaty H, Close JCT: Neuropsychological, physical, and functional mobility measures associated with falls in cognitively impaired older adults. J Gerontol A Biol Sci Med Sci 2014;69:987-995.

6 Van Der Wardt V, Logan P, Hood V, Booth V, Masud T, Harwood R: The association of specific executive functions and falls risk in people with mild cognitive impairment and early-stage dementia. Dement Geriatr Cogn Disord 2015;40:178-185.

7 Rockwood K, Fay S, Jarrett P, Asp E: Effect of galantamine on verbal repetition in AD: a secondary analysis of the VISTA trial. Neurology 2007;68:1116-1121.

8 Lopes P, Pino M, Carletti G, Hamidi S, Legué S, Kerhervé H, et al: Co-conception process of an innovative assistive device to track and find misplaced everyday objects for older adults with cognitive impairment: the TROUVE project. IRBM 2016;37:52-57.

9 Flicker C, Ferris SH, Reisberg B: Mild cognitive impairment in the elderly: predictors of dementia. Neurology 1991;41:1006-1009.

10 Hodges JR, Erzinçlioğlu S, Patterson K: Evolution of cognitive deficits and conversion to dementia in patients with mild cognitive impairment: a very-long-term follow-up study. Dement Geriatr Cogn Disord 2006;21: 380-391.

11 Johnson JK, Pa J, Boxer AL, Kramer JH, Freeman K, Yaffe K: Baseline predictors of clinical progression among patients with dysexecutive mild cognitive impairment. Dement Geriatr Cogn Disord 2010;30:344-351.

12 Bigelow RT, Semenov YR, Trevino C, Ferrucci L, Resnick SM, Simonsick EM, et al: Association between visuospatial ability and vestibular function in the Baltimore Longitudinal Study of Aging. J Am Geriatr Soc 2015;63: 1837-1844.

13 Popp P, Wulff M, Finke K, Rühl M, Brandt T, Dieterich M: Cognitive deficits in patients with a chronic vestibular failure. J Neurol 2017;264:554-563.

14 Kremmyda O, Hüfner K, Flanagin VL, Hamilton DA, Linn J, Strupp M, et al: Beyond dizziness: virtual navigation, spatial anxiety and hippocampal volume in bilateral vestibulopathy. Front Hum Neurosci 2016;10:139.

15 Stackman RW, Clark AS, Taube JS: Hippocampal spatial representations require vestibular input. Hippocampus 2002;12:291-303.

16 Xie Y, Bigelow RT, Frankenthaler SF, Studenski SA, Moffat SD, Agrawal Y: Vestibular loss in older adults is associated with impaired spatial navigation: data from the Triangle Completion Task. Front Neurol 2017;8: 173.

17 Yoder RM, Goebel EA, Köppen JR, Blankenship PA, Blackwell AA, Wallace DG: Otolithic information is required for homing in the mouse. Hippocampus 2015;25:890-899.

18 Yoder RM, Kirby SL: Otoconia-deficient mice show selective spatial deficits. Hippocampus 2014;24:11691177.

19 Blankenship PA, Cherep LA, Donaldson TN, Brockman SN, Trainer AD, Yoder RM, et al: Otolith dysfunction alters exploratory movement in mice. Behav Brain Res 2017;325:1-11.

20 Baek JH, Zheng Y, Darlington CL, Smith PF: Evidence that spatial memory deficits following bilateral vestibular deafferentation in rats are probably permanent. Neurobiol Learn Mem 2010;94:402-413.

21 Russell NA, Horii A, Smith PF, Darlington CL, Bilkey DK: Long-term effects of permanent vestibular lesions on hippocampal spatial firing. J Neurosci 2003;23:6490-6498.

22 Glasauer S, Amorim M-A, Viaud-Delmon I, Berthoz A: Differential effects of labyrinthine dysfunction on distance and direction during blindfolded walking of a triangular path. Exp Brain Res 2002;145:489-497.

23 Brandt T, Schautzer F, Hamilton DA, Brüning R, Markowitsch HJ, Kalla R, et al: Vestibular loss causes hippocampal atrophy and impaired spatial memory in humans. Brain 2005;128:2732-2741.

24 Harun A, Oh ES, Bigelow RT, Studenski S, Agrawal Y: Vestibular impairment in dementia. Otol Neurotol 2016; 37:1137-1142.

25 Nakamagoe K, Fujimiya S, Koganezawa T, Kadono K, Shimizu K, Fujizuka N, et al: Vestibular function impairment in Alzheimer's disease. J Alzheimers Dis 2015;47:185-196.

26 Previc FH: Vestibular loss as a contributor to Alzheimer's disease. Med Hypotheses 2013;80:360-367.

27 Wei EX, Oh ES, Harun A, Ehrenburg M, Agrawal Y: Vestibular loss predicts poorer spatial cognition in patients with Alzheimer's disease. J Alzheimers Dis 2018;61:995-1003.

28 McKhann GM, Knopman DS, Chertkow H, Hyman BT, Jack CR, Kawas CH, et al: The diagnosis of dementia due to Alzheimer's disease: recommendations from the National Institute on Aging-Alzheimer's Association workgroups on diagnostic guidelines for Alzheimer's disease. Alzheimers Dement 2011;7:263-269. 
Wei et al.: Saccular Impairment in Alzheimer's Disease Is Associated with Driving Difficulty

29 Albert MS, DeKosky ST, Dickson D, Dubois B, Feldman HH, Fox NC, et al: The diagnosis of mild cognitive impairment due to Alzheimer's disease: recommendations from the National Institute on Aging-Alzheimer's Association workgroups on diagnostic guidelines for Alzheimer's disease. Alzheimers Dement 2011;7:270279.

30 Perneczky R, Wagenpfeil S, Komossa K, Grimmer T, Diehl J, Kurz A: Mapping scores onto stages: Mini-Mental State Examination and Clinical Dementia Rating. Am J Geriatr Psychiatry 2006;14:139-144.

31 Li C, Zuniga MG, Nguyen KD, Carey JP, Agrawal Y: How to interpret latencies of cervical and ocular vestibularevoked myogenic potentials: our experience in fifty-three participants. Clin Otolaryngol 2014;39:297-301.

32 Li C, Layman AJ, Carey JP, Agrawal Y: Epidemiology of vestibular evoked myogenic potentials: data from the Baltimore Longitudinal Study of Aging. Clin Neurophysiol 2015;126:2207-2215.

33 Nguyen KD, Welgampola MS, Carey JP: Test-retest reliability and age-related characteristics of the ocular and cervical vestibular evoked myogenic potential tests. Otol Neurotol 2010;31:793-802.

34 Money J, Alexander D, Walker HT Jr: A Standardized Road Map Test of Direction Sense. Johns Hopkins Press, 1965.

35 Rainville C, Marchand N, Passini R: Performances of patients with a dementia of the Alzheimer type in the Standardized Road-Map Test of Direction Sense. Neuropsychologia 2002;40:567-573.

36 Mapstone M, Steffenella TM, Duffy CJ: A visuospatial variant of mild cognitive impairment: getting lost between aging and AD. Neurology 2003;60:802-808.

37 Cohen HS, Ewell LR, Jenkins HA: Disability in Meniere's disease. Arch Otolaryngol Head Neck Surg 1995;121: 29-33.

38 Inoue Y, Ogawa K, Kanzaki J: Quality of life of vestibular schwannoma patients after surgery. Acta Otolaryngol 2001;121:59-61.

39 Wei EX, Agrawal Y: Vestibular dysfunction and difficulty with driving: data from the 2001-2004 National Health and Nutrition Examination Surveys. Front Neurol 2017;8:557.

40 Cohen HS, Wells J, Kimball KT, Owsley C: Driving disability and dizziness. J Safety Res 2003;34:361-369.

41 Clack TD, Milburn WO, Graham MD: Ear-eye reflexes while riding in a car. Laryngoscope 1985;95:182-185.

42 MacDougall HG, Moore ST, Black RA, Jolly N, Curthoys IS: On-road assessment of driving performance in bilateral vestibular-deficient patients. Ann NY Acad Sci 2009;1164:413-418.

43 Drachman DA, Swearer JM: Driving and Alzheimer's disease: the risk of crashes. Neurology 1993;43:24482456.

44 Logsdon RG, Teri L, Larson EB: Driving and Alzheimer's disease. J Gen Intern Med 1992;7:583-588.

45 Dubinsky RM, Williamson A, Gray CS, Glatt SL: Driving in Alzheimer's disease. J Am Geriatr Soc 1992;40:11121116.

46 Anderson SW, Aksan N, Dawson JD, Uc EY, Johnson AM, Rizzo M: Neuropsychological assessment of driving safety risk in older adults with and without neurologic disease. J Clin Exp Neuropsychol 2012;34:895-905.

47 Anstey KJ, Horswill MS, Wood JM, Hatherly C: The role of cognitive and visual abilities as predictors in the Multifactorial Model of Driving Safety. Accid Anal Prev 2012;45:766-774.

48 Dawson JD, Uc EY, Anderson SW, Johnson AM, Rizzo M: Neuropsychological predictors of driving errors in older adults. J Am Geriatr Soc 2010;58:1090-1096.

49 Lundberg C, Hakamies-Blomqvist L, Almkvist O, Johansson K: Impairments of some cognitive functions are common in crash-involved older drivers. Accid Anal Prev 1998;30:371-377.

50 Ferreira IS, Simões MR, Marôco J: The Addenbrooke's Cognitive Examination Revised as a potential screening test for elderly drivers. Accid Anal Prev 2012;49:278-286.

51 Silva MT, Laks J, Engelhardt E: Neuropsychological tests and driving in dementia: a review of the recent literature. Rev Assoc Med Bras 2009;55:484-488.

52 Reger MA, Welsh RK, Watson GS, Cholerton B, Baker LD, Craft S: The relationship between neuropsychological functioning and driving ability in dementia: a meta-analysis. Neuropsychology 2004;18:85-93.

53 O'Keefe J, Nadel L: The Hippocampus as a Cognitive Map. Oxford, Clarendon Press, 1978.

54 Indovina I, Riccelli R, Chiarella G, Petrolo C, Augimeri A, Giofrè L, et al: Role of the insula and vestibular system in patients with chronic subjective dizziness: an fMRI study using sound-evoked vestibular stimulation. Front Behav Neurosci 2015;9:334.

55 Just KS, Schneider KL, Schurig M, Stingl JC, Brockmöller J: Falls: the adverse drug reaction of the elderly and the impact of pharmacogenetics. Pharmacogenomics 2017;18:1281-1297.

56 Nordell E, Kristinsdottir EK, Jarnlo GB, Magnusson M, Thorngren KG: Older patients with distal forearm fracture. A challenge to future fall and fracture prevention. Aging Clin Exp Res 2005;17:90-95.

57 Gleason CE, Gangnon RE, Fischer BL, Mahoney JE: Increased risk for falling associated with subtle cognitive impairment: secondary analysis of a randomized clinical trial. Dement Geriatr Cogn Disord 2009;27:557-563.

58 Willis J, Ramulu P: Poor vision and self-reported functional difficulties among recently hospitalized individuals in the United States: poor vision and its impact on visual function. Ophthalmic Epidemiol 2016;23: 154-161.

59 Algase DL, Son GR, Beattie E, Song JA, Leitsch S, Yao L: The interrelatedness of wandering and wayfinding in a community sample of persons with dementia. Dement Geriatr Cogn Disord 2004;17:231-239. 